\title{
Développement et validation d'un plan de soins de suivi (PSS) pour des femmes atteintes du cancer de l'endomètre lors de la transition de la fin du traitement actif vers la survie au cancer
}

par Johanne Hébert et Lise Fillion

\section{RESUME}

De nombreux survivants du cancer terminent leur traitement sans connaître les risques associés pour la santé et sont peu préparés pour gérer leurs besoins de santé dans la phase de survie. De plus, les guides de pratique pour les soins de suivi ne sont pas disponibles et les besoins psychosociaux des survivants sont souvent peu évalués. Le développement et la mise en place d'un plan de soins de suivi personnalisé à la fin du traitement actif pour des femmes atteintes du cancer de l'endomètre (FACE) est proposé pour répondre au besoin d'information et faciliter la transition.

Problématique : La phase de l'après-traitement est une phase distincte et encore négligée du continuum de soins en oncologie. Cette phase correspond à une transition entre deux phases de la trajectoire de soins, traitement et survie qui présente de nombreux défis pour les personnes survivantes, les soignants et le système de santé.

Objectif : Visant à faciliter la transition entre la fin du traitement actif et la phase de survie au cancer, deux objectifs sont poursuivis : 1) Développer un plan de soins de suivi (PSS) à partir de la recension des écrits et selon la perspective de FACE, de professionnels de la santé impliqués avec la clientèle cible et de gestionnaires de proximité en oncologie et 2) Valider le PSS auprès de l'équipe interdisciplinaire.

Méthode : Pour le premier objectif, les besoins de FACE lors de la fin du traitement actif (fin immédiate, 3 mois et 6 mois) de même que les perceptions de professionnels de la santé et de gestionnaires de proximité ont été recueillies par entrevues et groupe de discussion concernant l'utilité d'un PSS, son contenu et son format souhaitable. Une analyse de contenu des données d'entrevues a été réalisée

\section{AU SUJET DES AUTEURES}

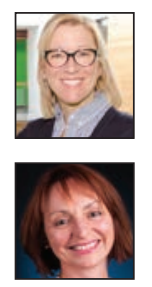

Johanne Hébert, inf., M.Sc., Ph. D(c), Professeure, Département des sciences infirmières, Université du Québec à Rimouski, UQA

Lise Fillion, inf., Ph. D, Professeure titulaire, Faculté des sciences infirmières, Université Laval

Correspondance : Johanne Hébert, inf, M.Sc., Ph. D(c),

Professeure, Département des sciences infirmières, Université du Québec à Rimouski, campus de Lévis, 1595, boulevard AlphonseDesjardins, Lévis (Québec) G6V 0A6

418 833-8800 ext.3243 ; e-mail : johanne_hebert@uqar.ca

DOI: $10.5737 / 236880762712232$ selon l'approche de Miles Q Huberman (2003) Pour le second objectif, un processus itératif de consultation avec les professionnels de la santé a permis l'atteinte d'une validation par consensus. Ces deux objectifs constituent la première phase qualitative d'un devis exploratoire séquentiel mixte permettant l'élaboration d'un PSS. La seconde phase a permis létude de la faisabilité de la mise en place du PSS lors de la transition de la fin du traitement actif vers la survie au cancer et fait l'objet d'un second article.

Résultats : Les entrevues ( $n=47)$ ont révélé un manque de préparation à la transition de la fin du traitement actif vers la survie au cancer chez les FACE. Les besoins d'information (80\%), de soutien émotionnel, notamment pour faire face à la peur de récidive (75\%), de gestion des symptômes physiques (45\%), et de soutien pour l'adaptation aux changements (45\%) sont précisés. Les données recueillies auprès de professionnels de la santé et de gestionnaires de proximité soutiennent l'utilité du PSS pour répondre à ces besoins. La démarche de validation par itération au sein de l'équipe interdisciplinaire permet d'obtenir un consensus sur le format et le contenu. La version finale du PSS est perçu comme un outil d'information et de communication pour la phase de survie. Des barrières concernant son transfert à la pratique clinique sont toutefois rapportées.

Conclusion : Cette étude présente l'ensemble de la démarche permettant l'élaboration d'un PSS qui intègre à la fois les besoins des survivantes du cancer de l'endomètre et les avis des professionnels de la santé qui dispensent des soins et gestionnaires de proximité en oncologie qui organisent les soins pour cette clientèle. Des indications sur la façon dont le PSS pourrait être mis en place au sein de cette organisation sont formulées.

Mots clés : transition, fin du traitement actif, besoins, survie au cancer, plan de soins de suivi

A u Canada, les cancers gynécologiques représentent 12\% Ades nouveaux cancers chez les femmes ; parmi eux, le cancer de l'endomètre est le plus fréquent et représente le quatrième cancer le plus courant chez la femme (Société Canadienne du cancer, [SCC] 2015). Bien que l'incidence augmente de 2,6\% depuis 2004, l'amélioration de la prévention, $\mathrm{du}$ dépistage et des traitements favorisent la survie relative du cancer de l'endomètre et dépasse les 80\% (SCC, 2015).

La fin du traitement actif du cancer et le début de la survie constituent une phase distincte et négligée du continuum de soins. Cette phase correspond à une période de transition (entre la phase de traitements et la phase de survie) qui 
présente de nombreux défis pour la personne survivante, les soignants et le système de santé (Hewitt, Greenfield et Stovall, 2006 ; Jones et Grunfeld, 2011). Pour la personne survivante, la fin du traitement actif correspond souvent à une transition complexe, tant au niveau du continuum de soins (entre traitement et survie) qu'au niveau personnel, pouvant représenter alors un événement de vie majeur. Bien que la fin du traitement soit généralement vécue comme un événement positif, un nombre croissant d'études documentent les difficultés liées à cette phase du continuum de soins (Beesley et al., 2008; Jones et al., 2012). Les conséquences du cancer et de ses traitements amènent des défis dans les domaines physique, informationnel, émotionnel, psychologique, social, pratique et spirituel et peuvent compromettre la qualité de vie (Fitch, Porter \& Page, 2008 ; Hewitt et al., 2006 ; Steele $\&$ Fitch, 2008). Pour les soignants et le système de santé, la diversité des personnes, des diagnostics, des traitements et des séquelles potentielles liées augmente la complexité des soins à la phase de survie (Dahl, Wittrup, Vaeggemose, Petersen \& Blaakaer, 2013). Cette complexité est accrue par le manque de lignes directrices sur l'orientation des soins de survie au cancer (Landier, 2009).

Des études suggèrent que les femmes atteintes du cancer de l'endomètre ne se sentent pas préparées pour la phase de l'après-traitement et la survie au cancer (Jones et al., 2012 ; Nicolaije et al., 2012). Plusieurs besoins (physiques, informationnels et psychosociaux) demeurent insatisfaits chez ces survivantes (Beesley et al., 2008 ; Nicolaije et al., 2012 ; Steele \& Fitch, 2008). De fait, des besoins physiques et des effets secondaires persistants et tardifs sont rapportés (Abbott-Anderson \& Kwekkeboom, 2012; Jones et al., 2012; Vaz et al., 2011; Vollrath, Zenger, Singer, Einenkel \& Hinz, 2013). La difficulté à gérer des symptômes physiques comme la fatigue, la douleur, des troubles du sommeil et des problèmes cognitifs est décrite par Wu et Harden (2015).

De plus, plusieurs études ont documenté les besoins d'information à la fin du traitement actif concernant la prévention et les risques de récidive, la possibilité de seconds cancers et d'autres problèmes de santé liés aux traitements et à la maladie, les effets secondaires persistants et tardifs des traitements sur les plans physique et psychosocial et les ressources psychosociales (Brennan, Butow, Spillane \& Boyle, 2010 ; Greimel, Lahousen, Dorfer, Lambauer \& Lang, 2011 ; Nicolaije et al., 2012 ; Papadakos et al., 2012). Les survivantes de cancers gynécologiques décrivent d'ailleurs des difficultés d'accessibilité à l'information et aux ressources, ainsi que de la difficulté à communiquer avec leurs professionnels de la santé (Jones et al., 2012 ; Nicolaije et al., 2012).

Les cancers gynécologiques et leurs traitements auraient également des répercussions sur les besoins psychosociaux tout au long du continuum de soins. Sur le plan psychologique, les préoccupations liées à la récidive de la maladie, aux effets secondaires persistants et tardifs et à la possibilité d'une survie limitée sont communes (Dahl et al., 2013; Simard et al., 2013). La peur de récidive du cancer (PRC) se classe parmi les problèmes les plus fréquemment rapportés chez tous les survivants du cancer (Armes et al., 2009; Jefford et al., 2008;
Simard et al., 2013) et chez la majorité des femmes atteintes de cancers gynécologiques (Beesley et al., 2008; Dahl et al., 2013; Fitch \& Steele, 2010; Steele \& Fitch, 2008). Des études soutiennent l'association entre la PRC, la détresse, la dépression, l'anxiété et l'évitement/l'intrusion chez les survivants du cancer (Simard et al., 2013). La PRC est également associée à une plus grande fréquence ou un plus grand nombre de symptômes physiques (effets secondaires globaux, fatigue, douleur, image/apparence corporelle) chez les survivants (Simard et al., 2013).

Pour répondre aux besoins de la fin du traitement actif vers la survie au cancer, le rapport charnière de L'Institute of Medicine (IOM) « From cancer patient to cancer survivor : lost in transition » propose des recommandations pour les soins de suivi en survie au cancer. On y souligne que la phase de survie est une phase distincte et négligée du continuum de soins. Cette phase serait mal coordonnée et de nombreux survivants et soignants ne recevraient pas suffisamment d'information sur les soins de suivi, les effets tardifs et à long terme du cancer et les risques élevés du cancer et de ses traitements pour la santé à long terme (Hewitt et al., 2006). De fait, des études soulignent que la gestion des symptômes et le soutien aux survivants à cette phase demeurent fragmentés et mal coordonnés (Jefford et al., 2011; Sprague et al., 2013). L'accès à de l'information sur l'après-traitement (Jones et al., 2012; Beckjord et al., 2008) et le maintien des soins de soutien dans cette phase de la trajectoire de soins (Jones et al., 2012 ; Marbach \& Griffie, 2011) sont recommandés. De l'information et du soutien favorisant la réponse à des besoins spécifiques pourraient d'une part prévenir la détresse (Steele \& Fitch, 2008), contribuer à l'empowerment des FACE et faciliter ainsi le processus de transition (Meleis et al., 2000). L'information pertinente, le soutien pour le développement des compétences et de la confiance favorisent l'engagement de la personne survivante dans l'autogestion de ses soins (McCorkle et al., 2011).

Pour faciliter la réponse aux besoins d'information et de soutien, les modèles de soins centrés sur la personne reconnaissent l'importance de clarifier les rôles et responsabilités de chacun dans les soins de suivi en survie au cancer (Epstein \& Street, 2007). Cette clarification pourtant nécessaire à la coordination des soins demeure toutefois ambigüe et peu définie dans cette phase du continuum de soins (Aubin et al., 2011; Bober et al., 2009).

Permettant à la fois l'information, la communication, la clarification et la coordination des soins (Curcio, Lambe, Schneider \& Khan, 2012 ; Faul et al., 2012 ; Forsythe et al., 2013; McCorkle et al., 2011), la remise d'un plan de soins de suivi (PSS) à chaque personne qui termine son traitement actif du cancer est proposé comme outil clinique. Il s'agit d'ailleurs d'une des recommandations de l'IOM (Hewitt et al., 2006). Le PSS constituerait un élément de continuité de soins de qualité (Howell et al., 2011). Il constituerait un lien de partenariat entre la personne survivante et l'équipe de soins (McCorkle et al., 2011). Actuellement, peu de survivantes de cancers gynécologiques ont accès à un PSS (Brothers, Easley, Salani \& Andersen, 2013 ; Grover et al., 2012 ; Sabatino et al., 2013 ; Salani, 2013). 
Lors de l'élaboration d'un PSS, il est recommandé de prendre en compte à la fois les perspectives des personnes atteintes de cancer ciblées et les avis des membres de l'équipe interdisciplinaire impliquée (Baravelli et al., 2009 ; Burg, Lopez, Dailey, Keller \& Prendergast, 2009 ; McCorkle et al., 2011 ; Smith, Singh-Carlson, Downie, Payeur \& Wai, 2011). Pourtant peu d'études ont documenté le processus de développement d'un PSS intégrant la perception de ces acteurs. À notre connaissance, aucune n'a été réalisée dans le contexte québécois.

Cet article présente la première phase de l'étude doctorale de la première auteure. Les objectifs poursuivis sont le développement et la validation d'un PSS pour des femmes atteintes du cancer de l'endomètre avec traitements adjuvants.

\section{METHODOLOGIE}

\section{Devis et participants}

L'ensemble de la démarche doctorale repose sur un devis exploratoire séquentiel (Creswell \& Plano Clark, 2011). Ce devis comprend une première phase d'élaboration suivie d'une phase d'exploration de l'implantation, soit une étude de faisabilité. La première séquence qualitative présentée dans cet article, comprend deux objectifs, l'élaboration et la validation du PSS.

Pour répondre au premier objectif correspondant au développement d'un PSS pour des FACE, un échantillon intentionnel a été constitué et composé de deux groupes d'acteurs : 1) des femmes atteintes du cancer de l'endomètre avec traitements adjuvants et 2) des professionnels de la santé travaillant avec la population cible (gynéco-oncologues, radio-oncologues, hémato-oncologue, médecins généralistes de l'établissement et de l'extérieur, infirmières de l'équipe spécialisée et infirmières en radio-oncologie, psycho-oncologues, nutritionniste, travailleur social, kinésiologue) et des gestionnaires de proximité en oncologie (cliniciens et décideurs impliqués dans les soins en oncologie). Les critères d'inclusion pour les FACE étaient les suivants : a) être atteinte du cancer de l'endomètre et avoir reçu des traitements adjuvants (chimiothérapie/radiothérapie/curiethérapie) seuls ou combinés b) avoir terminé les traitements actifs du cancer et c) être âgée de 18 ans et plus. Les critères de sélection permettent d'assurer une diversité d'expériences et de tenir compte de trois moments précis de la période de transition (fin des traitements, suivi de 3 mois et suivi de 6 mois). Pour les deux autres groupes, le critère d'inclusion était d'être impliqué dans la dispense ou l'organisation des soins de la clientèle cible.
Pour répondre au second objectif de validation du PSS, la méthode Delphi (Brown, 1968) qui vise à obtenir un avis aussi consensuel que possible d'un groupe d'experts a été utilisée. Deux groupes de discussion focalisée ont été réalisés avec des participants du groupe des professionnels de la santé soient les infirmières et les médecins spécialistes afin de permettre la validation du contenu de l'outil (PSS) et du format proposé.

\section{Procédure pour les FACE}

L'infirmière pivot en oncologie (IPO) de l'équipe d'oncologie spécialisée en cancers gynécologiques a sélectionné les patientes éligibles puis les a informées de l'étude. Celles qui étaient intéressées à recevoir plus d'information sur cette étude ont été invitées à rencontrer l'étudiante-chercheure ou une assistante de recherche (AR) qui les a informés du protocole et les a invitées à participer. Celles qui ont accepté de participer ont complété un formulaire de consentement et ont participé à une entrevue semi-structurée d'une durée de 30 à 45 minutes. Létude a reçu l'approbation éthique du centre hospitalier universitaire dans lequel la recherche s'est déroulée.

\section{Collecte de données qualitatives}

Les données qualitatives ont été collectées auprès de tous les participants à l'étude par entrevues individuelles ou par groupes de discussion focalisés (Focus group). Des guides d'entrevues et de discussions ont été élaborés à partir des concepts centraux des modèles théoriques retenus pour l'étude doctorale, soient la théorie intermédiaire de la transition de Meleis, Sawyer, Im, Hilfinger Messias, et Schumacher (2000), le cadre des soins de soutien en oncologie (Fitch, 1994) et le modèle de Patton (1997), souvent utilisé lors d'études d'implantation d'une innovation en santé. La collecte de ces données visait la description des besoins globaux de FACE et les perceptions de professionnels de la santé et de gestionnaires de proximité sur l'utilité, le contenu et le format souhaitable d'un PSS permettant de répondre à ces besoins. L'ensemble des concepts clés et indicateurs, leur lien avec la théorie, et leur sélection selon le groupe d'acteurs ciblés est résumé au tableau 1. De façon concomitante, les bases de données PubMed, CINAHL, EMBASE and Cochrane Library ont été consultées avec les mots-clés suivants : «Survivorship care plans, follow-up care, cancer care plan, cancer survivorship care, survivorship care planning, cancer treatment summary, post-treatment cancer care, cancer transitions, cancer survivors, gynecologic cancer survivors ». De plus, de nombreux

Tableau 1 : Concepts-clés et indicateurs retenus pour élaborer le contenu du PSS

\begin{tabular}{|l|l|l|l|}
\hline Groupes d'acteurs & Concepts & Indicateur/Questionnaire & Théorie/Modèle \\
\hline FACE & $\begin{array}{l}\text { Transition /Besoins } \\
\text { Peur de récidive } \\
\text { Empowerment }\end{array}$ & $\begin{array}{l}\text { Besoins (SUNS) } \\
\text { Détresse (IPRC) } \\
\text { Empowerment (HeiQ) }\end{array}$ & $\begin{array}{l}\text { Meleis et al. (2000) et } \\
\text { Fitch, (1994) }\end{array}$ \\
\hline $\begin{array}{l}\text { Professionnels/ } \\
\text { Gestionnaires de } \\
\text { proximité }\end{array}$ & $\begin{array}{l}\text { Ressources } \\
\text { Effets du PSS } \\
\text { Impact du PSS }\end{array}$ & $\begin{array}{l}\text { But, objectif et ressources à mettre en place pour le PSS } \\
\text { Effet du PSS sur les FACE et autres professionnels } \\
\text { Impact global de la mise en œuvre }\end{array}$ & $\begin{array}{l}\text { Patton (1997) adapté par } \\
\text { Fillion et al., (2011) }\end{array}$ \\
\hline
\end{tabular}

Note. SUNS = Survivors Unmet Needs Survey, (Campbell et al., 2011); IPRC = Inventaire de la peur de récidive du cancer, (Simard \& Savard, 2009); HeiQ = Health Education Impact Questionnaire (Osborne et al., 2007). 
sites internet d'organisations en cancer tels que Princess Margaret Cancer Centre, National Cancer Institute (NCI), Canadian and American Cancer Society, American Society of Clinical Oncology (ASCO), LIVESTRONG Foundation, Society of Gynecologic Oncology (SGO), Journey Forward, Australian Cancer Survivorship Centre, Macmillan Cancer Support, Cancer Council Victoria ont été consultés pour leur pertinence avec le sujet de l'étude.

\section{Analyses des données}

Des données statistiques descriptives décrivent l'échantillon (variables socio démographiques et cliniques). L'analyse des données qualitatives a été réalisée selon l'approche de Miles et Huberman (2003) en trois étapes : 1) condensation des données (organisation des données), 2) présentation (assemblage organisée de l'information tirée des données) et 3) élaboration/vérification des données (conclusions finales de l'ensemble des données). Les entrevues individuelles et de groupes ont été audio-enregistrées sur support numérique et retranscrites intégralement. Suite à la transcription intégrale du verbatim et à sa lecture approfondie, plusieurs thèmes et catégories ont émergés et se regroupent en thématiques selon une liste de départ de codes provenant des cadres conceptuels de l'étude et des variables clés présentés au tableau 2. Les données ont été organisées et codifiées à l'aide du logiciel NVivo10. Une définition opérationnelle pour chacun des codes a été établie. L'interprétation de ces thématiques visait d'abord à décrire et à préciser les besoins de la population cible et ensuite, à décrire les perceptions et les attentes sur le contenu, l'utilité et le format souhaitable d'un PSS à la fin du traitement actif du cancer par l'ensemble des participants.

Pour assurer la crédibilité et la fiabilité de l'étude, la triangulation des méthodes de collectes des données a été utilisée. En plus des données qualitatives recueillies par entrevues individuelles, des données provenant d'études sélectionnées ont été ajoutées pour la compréhension plus complète du processus de développement (contenu et format) de PSS existants. De plus, une validation de la démarche de codification a été effectuée entre la chercheure principale et une équipe de chercheurs indépendante jusqu'à ce qu'un consensus soit établi pour la définition opérationnelle de chaque code (Miles \& Huberman, 2003).

\section{Élaboration d'un PSS}

À la suite de l'analyse de l'ensemble des données (entrevues et littérature), une première ébauche d'un PSS a été

Tableau 2 : Cadre méthodologique des données qualitatives

Meleis et al. (2000); Fitch (1994)

FACE

\section{Nature de la transition}

Prise de conscience, niveau d'engagement, changement et différence, espace dans le temps, points critiques et événements

\section{Besoins en période de survie}

Physiques, informationnels, émotionnels, psychologiques, sociaux, spirituels, pratiques

Conditions de transition facilitantes et entravantes

Sens donné à l'événement, croyances et attitudes, statut socio-

économique, ressources communautaires, préparation et connaissances

\section{Adaptation aux changements}

Stratégies d'adaptation actives-passives, préparation à

l'après-traitement

\section{Indicateurs de processus}

Sentiment d'être en lien, interaction avec les autres et l'environnement, situation dans le temps et l'espace, développement de la confiance et du coping

\section{Indicateurs de résultats}

Maîtrise des habiletés et comportements pour la gestion de la transition, renouvellement de l'identité, comportement de santé
Patton (1997) adapté par Fillion et al. (2011)

Professionnels de la santé et gestionnaires de proximité

Situation initiale et ressources nécessaires à la mise en place du PSS But, besoins, objectifs du PSS, justification et pertinence du PSS, crédibilité de la démarche

Activités réalisées avec les ressources disponibles

Rôles, ressources nécessaires pour la mise en place du PSS à la fin du traitement, outils cliniques et organisationnels disponibles

Réaction des participants à la mise en place du PSS

Espérance, avantages, difficultés et inconvénients à mettre en place le PSS

Participants s'engagent dans la mise en place du PSS et dans les activités

Mise en œuvre du PSS, implication de la part des infirmières et des membres de l'équipe interdisciplinaire

Effets du PSS sur la personne en transition/empowerment pour la survie

Évaluer et répondre aux besoins ; faciliter les soins centrés sur la personne ;

Améliorer connaissances des ressources, le soutien perçu, la satisfaction ;

Accroître la qualité des soins ; services ou ressources fournies dans le temps.

Effets sur la continuité des soins, les pratiques et les changements de comportements

Impact du PSS sur le rôle infirmier, nouvelles pratiques, interactions entre les membres de l'équipe et la première ligne

L'impact global intentionnel et non intentionnel

Importance et conséquences de la mise en œuvre, rôle infirmier, satisfaction et qualité de vie dans une perspective à long terme 


\begin{tabular}{|c|c|c|}
\hline \multicolumn{3}{|c|}{$\begin{array}{l}\text { Tableau } 3 \text { : Caractéristiques socio démographiques et médicales } \\
\text { des FACE }\end{array}$} \\
\hline Caractéristique & Participant & $\%$ \\
\hline \multicolumn{3}{|l|}{$\hat{A} g e(n=19)$} \\
\hline $44-60$ ans & 9 & 47.4 \\
\hline 61 ans et + & 10 & 52.6 \\
\hline \multicolumn{3}{|l|}{ État civil $(n=18)$} \\
\hline Célibataire & 3 & 16.7 \\
\hline Mariée & 8 & 44.4 \\
\hline Union libre & 4 & 22.2 \\
\hline Veuve & 3 & 16.7 \\
\hline \multicolumn{3}{|l|}{ Niveau d'éducation $(n=18)$} \\
\hline Primaire & 2 & 11.1 \\
\hline Secondaire & 4 & 22.2 \\
\hline Diplôme professionnel & 3 & 16.7 \\
\hline CEGEP & 1 & 5.6 \\
\hline Universitaire & 8 & 44.4 \\
\hline \multicolumn{3}{|l|}{ Statut d'emploi $(n=18)$} \\
\hline Temps plein & 3 & 16.7 \\
\hline Temps partiel & 1 & 5.6 \\
\hline Sans emploi & 1 & 5.6 \\
\hline Maladie & 3 & 16.7 \\
\hline Retraitée & 10 & 55.6 \\
\hline Md famille $(n=19)$ & 18 & 94.7 \\
\hline \multicolumn{3}{|l|}{ Traitements reçus $(n=20)$} \\
\hline Chimiothérapie seulement & 2 & 10.0 \\
\hline Chimiothérapie et curiethérapie & 3 & 15.0 \\
\hline Radiothérapie et curiethérapie & 5 & 25.0 \\
\hline Curiethérapie seulement & 2 & 10.0 \\
\hline $\begin{array}{l}\text { Chimiothérapie et radiothérapie et } \\
\text { curiethérapie }\end{array}$ & 8 & 40.0 \\
\hline
\end{tabular}

réalisée par l'étudiante chercheure. Une validation du contenu inspirée de la méthode Delphi (Brown, 1968) a été effectuée auprès des membres de l'équipe interdisciplinaire de la clientèle à l'étude. Un processus de consultation des membres de cette équipe selon une démarche itérative a été mis en place. La démarche de consultation s'est poursuivie jusqu'à l'obtention d'un consensus sur le contenu et le format d'un PSS à mettre en œuvre dans cette organisation auprès de cette clientèle.

\section{RESULTATS}

Les caractéristiques de l'échantillon sont d'abord présentées. Puis les résultats sont présentés autour des thèmes émergeants pour chaque groupe d'acteurs. La description du processus de consultation pour la validation de contenu et lélaboration du PSS termine cette démarche.

Tableau 4 : Cinq thèmes permettant de regrouper les besoins exprimés par les FACE

\begin{tabular}{|c|c|}
\hline Thèmes & Verbatim \\
\hline 1. Information & $\begin{array}{l}\text { "Je suis partie, puis je ne savais rien...je savais que } \\
\text { j'avais rendez-vous dans } 3 \text { mois avec l'oncologue, } \\
\text { puis dans } 6 \text { mois avec le radiologue " (FACE 19) } \\
\text { "J'aurais aimé savoir qu'il y avait des groupes de } \\
\text { soutien pour la peur de la récidive et pour nous } \\
\text { préparer à ce qui arrive après... tu te le fais dire, } \\
\text { mais t'as tellement d'émotion, puis de choses à } \\
\text { gérer " (FACE 12) } \\
\text { "J'ai un peu de colère car j'ai su il y a } 3 \text { semaines, } \\
\text { mon grade et mon stade de cancer qui ne m'avait } \\
\text { jamais été dit et que je ne savais même pas que ça } \\
\text { existait " (FACE 12). }\end{array}$ \\
\hline $\begin{array}{l}\text { 2. Soutien } \\
\text { émotionnel } \\
\text { face à la peur } \\
\text { d'une récidive }\end{array}$ & $\begin{array}{l}\text { "Des fois, je file tellement mal...puis tu te } \\
\text { demandes si ça ne se serait pas infiltré en quelque } \\
\text { part d'autre " (FACE 8) } \\
\text { " Mon nuage noir, je pense que c'est ma tête qui } \\
\text { n'arrête pas de réfléchir à ça » (FACE 12) } \\
\text { " Il reste que le hamster tourne toujours...tu } \\
\text { as beau vouloir l'arrêter, ça ne se contrôle pas } \\
\text { comme ça » (FACE 9) }\end{array}$ \\
\hline $\begin{array}{l}\text { 3. Soutien } \\
\text { relationnel }\end{array}$ & $\begin{array}{l}\text { "Après les traitements, les gens ont l'impression } \\
\text { que l'on est guéri. Il devient alors plus difficile d'en } \\
\text { parler. On a l'impression que les gens ont besoin } \\
\text { que l'on passe à un autre appel » (FACE 6) } \\
\text { "J'avais } 2 \text { madame qui avaient eu le cancer que } \\
\text { j'appelais et qui me disaient : ah oui, ça c'est bien } \\
\text { normal » (FACE 7) } \\
\text { "C'est tellement important de parler à quelqu'un } \\
\text { qui n'a pas peur de ce que je vais dire " (FACE 2) }\end{array}$ \\
\hline
\end{tabular}

4. Gestion des "C'est comme si, au lieu d'avoir 24 heures dans symptômes ma journée, j'en avais la moitié...tant mes énergies physiques sont diminuées " (FACE 6)

persistants "La fatigue, quand est-ce que ça va partir? Est-ce que ça va revenir comme avant? les selles, ça va tu revenir comme avant? ç'a pas de sens le ventre me grossit comme ç'a pas d'allure" (FACE19)

\begin{tabular}{l|l}
$\begin{array}{l}\text { 5. Adaptation } \\
\text { aux } \\
\text { changements }\end{array}$ & $\begin{array}{l}\text { "C'est comme s'il fallait que je ré-enligne ma vie } \\
\text { pour que cette nouvelle vie-là soit à l'image de } \\
\text { ce que j'ai vraiment le goût de vivre maintenant " } \\
\text { (FACE 6) } \\
\text { "Je suis en reconstruction... j’ai pris un an à me } \\
\text { reconstruire, mais là, c'est ma reconstruction } \\
\text { sociale qui est à refaire, à peaufiner " (FACE 12) }\end{array}$ \\
\hline
\end{tabular}

Léchantillon est constitué de 19 FACE : neuf lors du dernier traitement (T0), quatre lors du suivi de 3 mois (T1) et six lors du suivi de 6 mois (T2), 24 professionnels de la santé (cinq infirmières, neuf médecins spécialistes, cinq médecins généralistes, deux psycho-oncologues, une nutritionniste, un travailleur social, un kinésiologue) et quatre gestionnaires de proximité en oncologie. Les caractéristiques sociodémographiques et médicales des FACE sont présentées au tableau 3. 


\section{Les besoins exprimés par la clientèle ciblée}

Pour l'ensemble des FACE, aux 3 temps de mesure, plusieurs besoins ont été rapportés. Les besoins ont été regroupés en cinq thèmes et illustrés par un verbatim représentatif et sont présentés au tableau 4. La majorité des participantes reconnaissent plusieurs besoins non satisfaits. Le premier thème correspond au besoin d'information. Plus de $80 \%$ ont exprimé des besoins d'information en rapportant un manque à la fin du traitement concernant le traitement et maladie et les symptômes à surveiller, les effets secondaires persistants, le suivi à venir et les ressources disponibles.

Près de 75\% ont exprimé un besoin émotionnel particulier d'être rassurées face à leurs craintes d'une récidive de cancer, ce qui correspond au second thème. Les FACE soutiennent avoir des inquiétudes que le cancer revienne et ne pas savoir quoi surveiller. Plusieurs parlent de sentir une « épée de Damoclès » au-dessus de leur tête.

Le troisième thème réfère au besoin de se sentir en relation avec les autres (besoin relationnel), professionnels de la santé et les proches après la fin du traitement. Ne pas se sentir délaissée après la fin du traitement a été rapporté par près de la moitié des participantes (49\%). Bien que le soutien des proches soit primordial, des professionnels comme l'infirmière pivot en oncologie (IPO) qui répond à des questions lors d'appels ou de rencontres ou le soutien des pairs par les groupes de soutien sont nommés.

Le quatrième thème réfère à des besoins physiques. Les FACE (45\%) ont rapporté un besoin de soutien pour la gestion de symptômes divers tels que de la fatigue des problèmes gastro-intestinaux, de la douleur, des neuropathies, des problèmes de sommeil et des problèmes sexuels qui persistent et affectent la qualité de vie. Enfin le cinquième thème est lié au processus d'adaptation. Les participantes (45\%) ont rapporté un besoin de soutien psychologique (professionnels de la santé, autres survivants du cancer) pour les aider à s'adapter aux changements psychologiques apportés par la maladie et les traitements en plus de la PRC. Parmi les autres thèmes rapportés par les FACE, notons que $40 \%$ ont discuté de l'importance de garder le contact avec le médecin de famille pendant et après la maladie et que $30 \%$ ont discuté de la difficulté de retour au travail en raison des symptômes physiques persistants.

\section{Perceptions des professionnels de la santé et gestionnaires de proximité}

Les perceptions des professionnels de la santé et des gestionnaires de proximité concernant le PSS sont présentées au tableau 5 . Ces résultats sont présentés par thèmes et composantes.

Le premier thème porte sur l'utilité du PSS. Il comprend trois composantes soient l'information, la continuité de l'information et le soutien aux FACE. On reconnait d'abord que les informations données pour l'après-traitement sont sommaires et irrégulières et dépendent des membres de l'équipe et de la personne qui termine son traitement. De nombreux participants (79\%) soutiennent que le PSS pourrait être un outil d'information pour les FACE, un résumé sur les effets secondaires persistants et les symptômes à surveiller (quoi), les contacts en cas de besoin (qui) et le suivi à venir (quand). D'autres (54\%) mentionnent que l'information contenue dans le PSS serait une référence écrite utile aux professionnels à l'extérieur du milieu spécialisé (notamment le médecin de famille), par exemple, pour connaître l'histoire de la période des traitements et les effets secondaires à surveiller. Ainsi, il permettrait une continuité de l'information dans le but d'améliorer la continuité des soins et pourrait favoriser la communication entre les professionnels. Le PSS, comme référence écrite, procurerait un soutien aux FACE et pourrait faciliter la transition de la fin du traitement actif vers la survie. La rencontre de remise à la fin du traitement serait rassurante et pourrait permettre de faire le point à ce moment du continuum de soins tout en responsabilisant la personne sur ce qu'elle doit surveiller pour la phase de l'après-traitement.

Le second thème porte sur les éléments de contenu du PSS. Il comprend l'ensemble des composantes proposées par l'IOM (Hewitt et al., 2006) qui sont rapportés par la majorité des participants. On note cependant que seulement $14 \%$ ont parlé d'inclure des informations sur les comportements de santé sains à adopter en survie au cancer. Des précisions sont apportées lors de la validation de contenu sur la quantité d'information (particularités de la maladie et des traitements et liste de symptômes). Les participants ont souligné que l'information doit être facile à retrouver et que trop d'information pourrait augmenter l'anxiété. De plus, la simplicité de l'information (termes simples) pour faciliter la compréhension est rapportée par les professionnels de la santé.

Enfin, en ce qui a trait au dernier thème concernant le format souhaitable, cinq composantes ressortent. Le PSS décrit est court, avec des cases et crochets favorisant la rapidité de complétion et il doit être facile d'utilisation pour les utilisateurs (document informatisé spécifié par plusieurs participants). Certains recommandent que le PSS soit personnalisé, c'està-dire qu'il devrait être adapté selon les besoins de chaque personne. D’autres préfèrent que le PSS ait une section générale et une section personnalisée. La majorité recommande un modèle de base standardisé avec un contenu adapté selon la personne et le site de cancer et des espaces blanc pour compléter l'information.

Lorsqu'interrogés sur la personne la mieux placée pour remettre le PSS et le moment de remise, $50 \%$ ont désigné l'IPO, $21 \%$ le médecin spécialiste et $11 \%$, d'autres professionnels de l'équipe de soins significatifs pour la personne. Quant à la remise, le dernier traitement $(21 \%)$ ou vers la fin du traitement $(18 \%)$ seraient souhaitables. Par ailleurs, des difficultés ont été soulevées concernant la valeur ajoutée du PSS pour les professionnels de la santé et l'organisation. Des barrières de temps, de ressources (personnel et financières) et de trajectoire de soins (logistique) notamment la faisabilité de rencontrer chaque patiente qui termine son traitement actif sont soulevées. Plusieurs questionnent la surcharge de travail (temps) à remplir (papier ou informatique) et à remettre le PSS. Concernant les ressources, les notions de responsabilité (qui va faire quoi) et d'interdisciplinarité (comment partager la responsabilité) sont soulevées. 


\begin{tabular}{|c|c|c|}
\hline Thèmes et composantes & Participants (\%) & Verbatim \\
\hline \multicolumn{3}{|l|}{ 1.Utilité du PSS } \\
\hline $\begin{array}{l}\text { Informer FACE et autres } \\
\text { professionnels }\end{array}$ & 64 & $\begin{array}{l}\text { "Je vois vraiment que le but est } 2 \text { choses : que le patient ait son information, et que le médecin qui va suivre } \\
\text { le patient, mettons, à Blanc-Sablon après, ait l'information » (Prof 16) } \\
\text { "Surtout pour les professionnels de la santé qui comme moi, apprennent à connaître les effets secondaires } \\
\text { et les effets tardifs " (Prof 21) }\end{array}$ \\
\hline $\begin{array}{l}\text { Améliorer la continuité } \\
\text { des soins }\end{array}$ & 53 & $\begin{array}{l}\text { "Si le plan de suivi est bien précisé, organisé puis que ceux qui font la suite sont outillés en termes de } \\
\text { qu'est-ce que je devrais surveiller, à quel rythme, à quelle fréquence, pourquoi, quand est-ce que je réfère } \\
\text { au spécialiste " (Prof 8) }\end{array}$ \\
\hline $\begin{array}{l}\text { Favoriser la communication } \\
\text { entre professionnels }\end{array}$ & 50 & $\begin{array}{l}\text { "Faciliter la communication entre les différents intervenants à l'extérieur de nos murs parce que nous, on } \\
\text { fait énormément de gestion de symptômes par téléphone, des patients qui ont terminé leur traitement " } \\
\text { (Prof 7) }\end{array}$ \\
\hline $\begin{array}{l}\text { Faciliter la transition de la } \\
\text { fin du traitement }\end{array}$ & 32 & $\begin{array}{l}\text { "Qu'il y ait une intervention d’emblée, que les gens soient en mesure de savoir vers quoi et vers où se } \\
\text { diriger » (Prof 9) } \\
\text { « Le plan de suivi ça permet de faire le point avec le patient, à un moment clé de la trajectoire » (Prof 3) }\end{array}$ \\
\hline Rassurer & 25 & « Je le vois comme un outil de réassurance pour le patient » (Prof 10) \\
\hline Favoriser l'empowerment & 25 & "Un plan de match parce que les gens aiment avoir le contrôle de leur vie, de leur situation » (Prof 20) \\
\hline \multicolumn{3}{|l|}{ 2. Contenu du PSS } \\
\hline Coordination (qui fait quoi) & 54 & $\begin{array}{l}\text { «Pour votre hypertension, votre thyroide, c'est votre Md de famille...clarifier les rôles de chacun pour le } \\
\text { patient » (Prof 1) } \\
\text { «Les coordonnées, s'il se passe quelque chose, je m'en vais où, qu'est-ce que je fais? » (Prof 16) }\end{array}$ \\
\hline $\begin{array}{l}\text { Diagnostic et résumé de } \\
\text { traitement }\end{array}$ & 43 & $\begin{array}{l}\text { "Diagnostic, résumé de traitements parce que s'ils ont des problèmes et qu'ils arrivent dans d'autres milieux, } \\
\text { ça va permettre de faire des liens " (Prof } 3 \text { ) }\end{array}$ \\
\hline Information standardisée & 43 & $\begin{array}{l}\text { "Je pense qu'on est mieux, avec un « template » (Prof 37) } \\
\text { «Qu'il y ait quelque chose qui sorte automatiquement via la base informatique SICTO » (Prof 1) }\end{array}$ \\
\hline $\begin{array}{l}\text { Effets secondaires } \\
\text { persistants et tardifs }\end{array}$ & 40 & $\begin{array}{l}\text { "C'est quoi les raisons, lesquelles je devrais appeler mon infirmière pivot ou mon oncologue »(Prof 1) } \\
\text { «Qu'est-ce que j’ai à surveiller et qu'est-ce qui n'est pas nécessairement inquiétant »(Prof 12) }\end{array}$ \\
\hline Ressources & 40 & «Savoir qu'y a des ressources qui existent » (Prof 9) \\
\hline Autres services fournis & 36 & $\begin{array}{l}\text { "Le résumé des autres professionnels psycho-sociaux vus...s'ils ont vu la TS, l'ergothérapeute, l'infirmière, le } \\
\text { psychiatre ou la psychologue... au moins, qu'il y ait leur nom, leurs coordonnées " (Prof 10) }\end{array}$ \\
\hline \multicolumn{3}{|l|}{ 3. Format souhaité } \\
\hline Court & 57 & «Avoir un minimum d'informations pour les rassurer, mais tout en dosant en n'en mettant pas trop» (Prof 10) \\
\hline Cases et crochets & 43 & $\begin{array}{l}\text { "C'est sûr qu'un format à cocher...juste faire un crochet c'est toujours agréable » (Prof 10) } \\
\text { « Le plus coché possible tout en restant significatif »(Prof 12) }\end{array}$ \\
\hline $\begin{array}{l}\text { Rapide et simple } \\
\text { d'utilisation }\end{array}$ & 43 & « Rapide à consulter, rapide à compléter » (Prof 17) \\
\hline Personnalisé & 36 & $\begin{array}{l}\text { "Les besoins vont être probablement très différents en fonction des traitements, du diagnostic et de la } \\
\text { personnalité des patients » (Prof 1) } \\
\text { «L'information plus personnalisée par rapport au patient c'est quelque chose qui est facile à compléter » (Prof 3) }\end{array}$ \\
\hline Standardisé & 25 & «Il y a plusieurs choses qui peuvent être « printées » à l'avance, déjà intégrées » (Prof 1) \\
\hline
\end{tabular}




\section{Validation et présentation du PSS final}

L'intégration de l'ensemble des recommandations formulées par les deux groupes d'acteurs lors des entrevues et de la littérature a permis la première ébauche d'un PSS. Lébauche a été soumise à un processus de validation. Une démarche participative et itérative a été réalisée en deux étapes : 1) présentation du PSS pour validation à deux groupes d'acteurs (médecins spécialistes et infirmières) selon une démarche consensuelle pour chacun des items proposés dans le PSS, et 2) rencontres avec les IPO pour la mise au point et le pilotage du PSS informatisé. Ce processus de validation a nécessité trois rencontres de groupes, et six rencontres individuelles représentant un total de 20 heures pour permettre l'élaboration d'une version finale du PSS. D'abord, trois groupes de discussion focalisée d'une durée d'une heure ont été réalisés avec des participants du groupe des professionnels de la santé, soient les IPO $(\mathrm{n}=2)$ et les médecins spécialistes $(\mathrm{n}=8)$. Chaque item du PSS a été présenté par PowerPoint, afin de permettre la validation du contenu de l'outil (PSS) et du format proposé. Ensuite, les IPO impliquées dans l'implantation ont été rencontrées à six reprises pendant 90 minutes pour la mise en œuvre des recommandations à inclure dans le PSS et la mise au point technique de l'outil.

Le PSS final (version quatre) contient les éléments suivants : 1) informations personnelles, 2) résumé du traitement : type de cancer, stade et grade, chirurgie (date, type, particularités, chirurgien), types de traitements reçus : chimiothérapie (médicaments, cycles, particularités, dates de début et de fin, lieu du traitement); radiothérapie (région, dose totale, nombre de fractions, particularités, radio-oncologue, dates de début et de fin, lieu du traitement); curiethérapie (technique, dose totale, particularités, radio-oncologue, dates de début et de fin, lieu de traitement); thérapie hormonale (médicaments, dose, particularités, dates de début et de fin); fin de traitement (date de fin du traitement adjuvant et toxicités liées au traitement), 3) effets secondaires à long terme possibles, 4) surveillance des symptômes à signaler au médecin, 5) examens et suivi recommandés par les spécialistes 6) suivi de santé global, 7) contacts, 8) ressources, 9) objectifs de santé personnels, et 10) recommandations pour des comportements de santé sains (exercice, nutrition, tabagisme, alcool, exposition aux ultraviolets). Plusieurs sections comprennent des menus déroulants et de l'information standardisée pour le cancer de l'endomètre pour faciliter le remplissage du PSS.

\section{DISCUSSION}

La phase I de l'étude doctorale comprenait deux objectifs. Le premier objectif visait l'élaboration d'un PSS qui prend en compte les besoins de femmes atteintes du cancer de l'endomètre (FACE) à la fin du traitement actif et la perception de professionnels de la santé et gestionnaires de proximité d'un PSS. Celui-ci est perçu comme un outil d'information et de communication nécessaire à mettre en œuvre pour faciliter la transition de la fin du traitement actif vers la survie. Le second objectif visait la validation de contenu. La démarche par itération a permis d'obtenir un consensus au sein de l'équipe interdisciplinaire.

\section{La prise en compte des besoins des FACE}

Loriginalité de ce PSS est de prendre en compte les besoins exprimés par les FACE. La précision de ces besoins vient appuyer les résultats d'autres études déplorant que les survivantes du cancer ne sont pas suffisamment préparées pour la survie au cancer (Burg et al., 2009 ; Jones et al., 2012). Nos résultats suggèrent que les FACE ont de nombreux besoins qui ne sont pas satisfaits et qui affectent leur qualité de vie. Plusieurs expriment la difficulté de revenir à une vie «normale » avec des symptômes physiques qui persistent et le manque d'information sur les symptômes à surveiller (ce qui est normal ou non) et le suivi recommandé pour l'après-traitement. Les résultats de notre étude s'ajoutent aux constats souvent déplorés sur le manque d'information persistant (Jones et al., 2012 ; Nicolaije et al., 2012). Le besoin de recevoir de l'information à la fin du traitement actif chez les femmes atteintes de cancers gynécologiques est pourtant bien documenté (Brennan et al., 2010 ; Burg et al., 2009 ; Marbach \& Griffie, 2011 ; Nicolaije et al., 2012 ; Papadakos et al., 2012). Les FACE de notre étude expriment des besoins d'informations concernant les risques de récidive, les effets secondaires persistants et tardifs des traitements sur les plans physique et psychosocial, le soutien psychosocial disponible, et d'autres problèmes de santé liés aux traitements et à la maladie.

La majorité des participantes de l'étude ont exprimé la peur que la maladie revienne et le sentiment d'avoir une « épée de Damoclès » au-dessus de la tête. Les besoins émotionnels et psychologiques, notamment les préoccupations liées à la récidive de la maladie, aux effets secondaires persistants et tardifs et à la possibilité d'une diminution de la durée de vie sont communs à cette phase (Dahl et al., 2013; Simard et al., 2013). Des études suggèrent que les survivants qui présentent un niveau élevé de peur de récidive sont plus susceptibles de développer des problèmes d'anxiété (Simard \& Savard, 2015). La PRC décrite par les FACE correspond au sentiment d'anxiété qui est fréquemment observé dans l'année qui suit la fin du traitement initial et est souvent associé à la peur de récidive de la maladie (Harrington et al., 2010; Salani, 2013). Enfin, des difficultés d'adaptation aux changements provoqués par la maladie et les traitements et à une nouvelle réalité de l'aprèstraitement sont rapportés par les FACE. Létude de Thorne et Stajduhar (2012) souligne la difficulté d'adaptation et la confusion des émotions (insécurité, vulnérabilité, sentiment de perte et d'abandon) à la fin du traitement qui amène de nouvelles inquiétudes concernant la santé physique, le bien-être émotionnel, les relations sociales, le travail et les ressources financières.

\section{La contribution des professionnels de santé}

Notre étude fait également ressortir comment les professionnels de la santé voient le PSS comme un outil d'information pour la personne survivante et les professionnels de la santé, notamment pour le médecin de famille. Il est perçu comme un outil de continuité de l'information qui pourrait améliorer la continuité des soins et la communication entre professionnels. On précise que le PSS serait un outil pour orienter les soins de soutien, permettant de 
renforcer la capacité des FACE à mieux répondre à leurs besoins. Développé en collaboration avec les FACE dans le but de les informer et de les engager dans l'autogestion de leur santé, le PSS contribuerait à une meilleure coordination des soins (McCorkle et al., 2011). L'impact sur l'empowerment des FACE pourrait faciliter la transition de la fin du traitement vers la survie et aurait un effet indirect de « rassurance ».

La contribution d'un PSS à améliorer la coordination des soins et la communication entre les soignants à cette phase de la trajectoire de soins a également été soulignée dans les écrits (Curcio et al., 2012; Hewitt et al., 2006; Jefford et al., 2011). Le PSS constituerait un élément de continuité et de soins de qualité (Howell et al., 2011) et améliorerait le standard de soins (Haq et al., 2013). La démarche retenue fournit un exemple d'approche interdisciplinaire pour le développement et la mise en place d'un PSS tel que recommandé dans les écrits (Howell et al., 2011 ; Jefford et al., 2011).

\section{La précision du contenu et du format d'un PSS et le moment de remise}

Nos résultats soutiennent les recommandations de l'IOM car tous les éléments de contenu suggérés s'y retrouvent. Selon Stricker et al. (2011), seulement 59\% des PSS existants contiennent le contenu proposé par l'IOM (Hewitt et al., 2006). En plus de ces recommandations, les participants suggèrent un langage simple, compréhensible (non médical) et l'information contenue dans le PSS devrait être pertinente pour la personne survivante (Burg et al., 2009). En ce sens, trop d'information générale (symptômes possibles d'une récidive ou des effets secondaires potentiels) peut provoquer de l'anxiété et dissuader les personnes d'utiliser le PSS (Haq et al., 2013). Des études suggèrent d'inclure une section portant sur les problèmes psychologiques, légaux et financiers (Baravelli et al., 2009). De plus, nos résultats précisent l'importance d'intégrer une forme de plan d'action en intégrant une section sur les personnes ressources à contacter pour répondre aux besoins spécifiques. Notamment, une personne responsable du suivi devrait être indiquée dans le PSS (Baravelli et al., 2009 ; Howell et al., 2011) pour faciliter la coordination de l'après-traitement. En ce sens, le PSS élaboré répond à une recommandation précisant que cet instrument doit être personnalisé et ne doit pas être un « one size fits all » (Brennan, Gormally, Butow, Boyle, $\&$ Spillane, 2014). De plus, en le remettant à la fin des traitements, il permet que la bonne information soit donnée au bon moment (Haq et al., 2013).

Concernant le format, le PSS devrait être court, rapide et simple d'utilisation. Notre étude suggère des sections personnalisées et d'autres standardisées au type de cancer. Un format électronique et une remise papier aux survivantes lors du dernier traitement sont préconisés. Comme soulevé dans d'autres études (Baravelli et al., 2009 ; Dulko et al., 2013), l'infirmière

\section{RÉFÉRENCES}

Abbott-Anderson, K., \& Kwekkeboom, K.L. (2012). A systematic review of sexual concerns reported by gynecological cancer survivors. Gynecologic Oncology, 124(3), 477-489. serait la professionnelle indiquée pour compléter et remettre le PSS près de la fin du traitement ou rapidement après (Mayer, Gerstel, Leak, \& Smith, 2012). Une rencontre de fin de traitement permettrait de normaliser la transition de la fin du traitement actif vers la survie, d'introduire le PSS comme outil d'information et de coordination, de répondre aux questions soulevées, d'orienter vers des ressources si nécessaires et d'encourager la transition vers le médecin de famille (Jefford et al., 2011). Les FACE soutiennent que le médecin de famille devrait être un professionnel engagé dans les soins tout au long de la trajectoire de soins, notamment dans les soins de survie. En ce sens, l'étude de Smith et al. (2011) suggère une transition planifiée entre spécialistes et médecin de famille pour favoriser la continuité des soins lors de la transition de la fin du traitement et la survie.

\section{Les barrières anticipées à sa mise en œuvre}

Les résultats de l'étude rapportent toutefois des barrières au développement et à l'implantation d'un PSS déjà rapportées dans d'autres études. De fait, la difficulté de préciser les rôles et responsabilités de chaque membre de l'équipe pour remplir et remettre le PSS est soulevée, le manque de temps pour le développement et la mise en place personnalisés et le manque de ressources (de personnel et financières) sont rapportés (Brennan et al., 2014 ; Dulko et al., 2013).

\section{CONCLUSION}

La démarche permet de décrire l'élaboration d'un PSS qui intègre à la fois les besoins des survivantes et des professionnels de la santé et gestionnaires de proximité. De plus, elle fournit des indications sur la façon dont le PSS pourrait être mis en place au sein de cette organisation. Son volet exploratoire précise par ailleurs les nombreux défis auxquels les survivantes du cancer de l'endomètre font face dans la transition de fin du traitement vers la survie au cancer. Des besoins d'information sur la surveillance et la gestion de divers symptômes physiques persistants et tardifs, des besoins de soutien émotionnel et d'informations concernant la peur de la récidive et des besoins de soutien pour s'adapter au retour à la vie après le cancer sont clarifiés. Le PSS apparaît d'abord comme un outil d'information pour la personne survivante. Son implantation pourrait faciliter la transition en facilitant la réponse à divers besoins, en contribuant à mieux gérer la peur de la récidive, et en favorisant l'empowerment par la précision de comportements souhaitables. Actuellement, peu de survivants du cancer ont accès à un tel PSS. La phase II de notre démarche propose une étude permettant de documenter la faisabilité et l'utilité de le rendre accessible dès la fin des traitements.

\section{CONFLITS D'INTERETS}

Les auteures n'ont aucun conflit d'intérêts potentiel.

Armes, J., Crowe, M., Colbourne, L., Morgan, H., Murrells, T., Oakley, C., et al. (2009). Patients' supportive care needs beyond the end of cancer treatment: A prospective, longitudinal survey. Journal of Clinical Oncology, 27(36), 6172-6179. 
Aubin, M., Vézina, L., Verreault, P., Fillion, L., Hudon, E., Lehman, F., ... Morin, D. (2011). Patient, Primary Care Physician and Specialist expectations of Primary Care Physician involvement in Cancer Care. Journal of General Internal Medicine, 27(1), 8-15.

Baravelli, C., Krishnasamy, M., Pezaro, C., Schofield, P., Lotfi-Jam, K., Rogers, M., ... Jefford, M. (2009). The views of bowel cancer survivors and health care professionals regarding survivorship care plans and post treatment follow up. Journal of Cancer Survivorship: Research and Practice, 3(2), 99-108. doi:10.1007/s1176400900861

Beckjord, E.B., Arora, N.K., McLaughlin, W., Oakley-Girvan, I., Hamilton, A.S., \& Hesse, B.W. (2008). Health-related information needs in a large and diverse sample of adult cancer survivors: implications for cancer care. Journal of Cancer Survivorship: Research and Practice, 2(3), 179-189.

Beesley, V., Eakin, E., Steginga, S., Aitken, J., Dunn, J., \& Battistutta, D. (2008). Unmet needs of gynaecological cancer survivors: implications for developing community support services. PsychoOncology, 17(4), 392-400.

Bober, S.L., Recklitis, C.J., Campbell, E.G., Park, E.R., Kutner, J.S., Najita, J.J., \& Diller, L. (2009). Caring for cancer survivors: A survey of primary care physicians. Cancer, 115(Suppl 18), 4409-4418.

Brennan, M.E., Butow, P., Spillane, A.J., \& Boyle, F.M. (2010). Survivorship care after breast cancer: follow-up practices of Australian health professionals and attitudes to a survivorship care plan. Asia-Pacific Journal of Clinical Oncology, 6(2), 116-125.

Brennan, M.E., Gormally, J.F., Butow, P., Boyle, F.M., \& Spillane, A.J. (2014). Survivorship care plans: a systematic review of care plan outcomes. British Journal of Cancer, 111(10), 1899-1908.

Brothers, B.M., Easley, A., Salani, R., \& Andersen, B.L. (2013). Do survivorship care plans impact patients' evaluations of care? A randomized evaluation with gynecologic oncology patients. Gynecologic Oncology, 129(3), 554-558.

Brown, B.B. (1968). Delphi Process: A methodology used for the elicitation of opinions of experts. Santa Monica, California: ASTME Vectors.

Burg, M.A., Lopez, E.D.S., Dailey, A., Keller, M.E., \& Prendergast, B. (2009). The potential of survivorship care plans in primary care follow-up of minority breast cancer patients. Journal of General Internal Medicine, 24(Suppl 2), S467-S471.

Campbell, S.H., Sanson-Fisher, R.,Turner, D., Hayward, L., Wang, X.S., \& Taylor-Brown, J. (2011). Psychometric properties of cancer survivors' unmet needs survey. Supportive Care in Cancer, 19(2), 221-230.

Creswell, J.W., \& Plano Clark, V.L. (2011). Designing and conducting mixed methods research. (2e éd.). Thousand Oaks, CA: SAGE Publications.

Curcio, K.R., Lambe, C., Schneider, S., \& Khan, K. (2012). Evaluation of a cancer survivorship protocol: transitioning patients to survivors. Clinical Journal of Oncology Nursing 16(4), 400-406.

Dahl, L., Wittrup, I., Vaeggemose, U., Petersen, L.K., \& Blaakaer, J. (2013). Life after gynecologic cancer- A review of patient's quality of life, needs, and preferences in regard to follow-up. International Journal of Gynecological Cancer, 23(2), 227-234.

Dulko, D., Pace, C.M., Dittus, K.L., Sprague, B.L., Pollack, L.A., Hawkins, N.A., \& Geller, B.M. (2013). Barriers and facilitators to implementing cancer survivorship care plans. Oncology Nursing Forum, 40(6), 575-580.

Epstein, R.M., \& Street, R.L. (2007). Patient-centered communication in cancer care: promoting healing and reducing suffering. National Cancer Institute (NCI). Bethesda, MD: NIH Publication.
Faul, L.A., Rivers, B., Shibata, D., Townsend, I., Cabrera, P., Quinn, G.P., \& Jacobsen, P.B. (2012). Survivorship care planning in colorectal cancer: Feedback from survivors \& providers. Journal of Psychosocial Oncology, 30(2), 198-216.

Fillion, L., Cook, S., Blais, M.C., Veillette, A.M., Aubin, M., de Serres, M., Rainville, F., ...Fournier, B. (2011). Implementation of screening distress with professional cancer navigators. Oncologie, 13, 277-289.

Fitch, M.I. (1994). Providing supportive care for individuals living with cancer. Toronto. Ontario Cancer Treatment and Research Foundation.

Fitch, M.I., Porter, H.B., \& Page, B.D. (2008). Supportive care framework: A foundation for person-centered care. Toronto, ON: Pappin Communications.

Fitch, M.I., \& Steele, R. (2010). Identifying supportive care needs of women with ovarian cancer. Canadian Oncology Nursing Journal, 20(2), 66-74.

Forsythe, L.P., Parry, C., Alfano, C.M., Kent, E.E., Leach, C.R., Haggstrom, D.A., ... Rowland, J.H. (2013). Use of survivorship care plans in the United States: associations with survivorship care. Journal of the National Cancer Institute, 105(20), 1579-1587.

Greimel, E., Lahousen, M., Dorfer, M., Lambauer, M., \& Lang, U. (2011). Patients' view of routine follow-up after gynecological cancer treatment. European Journal of Obstetrics \& Gynecology and Reproductive Biology, 159(1), 180-183.

Grover, S., Hill-Kayser, C., Vachani, C., Hampshire, K., DiLullo, G.A., \& Metz, J.M. (2012). Patient reported late effects of gynecological cancer treatment. Gynecologic Oncology, 124(3), 399-403.

Haq, R., Heus, L., Baker, N.A., Dastur, D., Leung, F-H., Leung, E., Li, B., Vu, K., \& Parsons, J.A. (2013). Designing a multifaceted survivorship care plan to meet the information and communication needs of breast cancer patients and their family physicians: results of a qualitative pilot study. BMC medical informatics and decision making, 13(76). doi 10.1186/1472-6947-13-76

Harrington, C.B., Hansen, J.A., Moskowitz, M., Todd, B.L., \& Feuerstein, M. (2010). It's not over when it's over: Long-term symptoms in cancer survivors-A systematic review. International Journal of Psychiatry in Medicine, 40(2), 163-181.

Hewitt, M., Greenfield, S., \& Stovall, E. (2006). From cancer patient to cancer survivor: Lost in transition. Washington DC: The National Academies Press.

Howell, D., Hack, T.F., Oliver, T.K., Chulak, T., Mayo, S., Aubin, M., ... Sinclair, S. (2011). Survivorship services for adult cancer population: A Pan-Canadian guideline. Current Oncology, 18(6), e265-e281.

Jefford, M., Lotfi-Jam, K., Baravelli, C., Grogan, S., Rogers, M., Krishnasamy, M., ...Schofield, P. (2011). Development and pilot testing of a nurse-led posttreatment support package for bowel cancer survivors. Cancer Nursing, 34(3), E1-E10.

Jones, J.M., Ferguson, S., Edwards, E., Walton, T., McCurdy, N., \& Howell, D. (2012). Experiences of care delivery: endometrial cancer survivors at end of treatment. Gynecologic Oncology, 124(3), 458-464.

Jones, J.M., \& Grunfeld, E. (2011). Specific challenges in optimizing health care for cancer survivors. In M. Feuerstein \& P.A. Ganz (Eds.), Health services for cancer survivors: Practice, policy and research (pp.3-25). New York: Springer.

Landier, W. (2009). Survivorship care: essential components and models of delivery. Oncology (Williston Park), 23(Suppl Nurse Ed), 46-53.

Marbach, T.J., \& Griffie, J. (2011). Patient preferences concerning treatment plans, survivorship care plans, education, and support services. Oncology Nursing Forum, 38(3), 335-342. 
Mayer, D.K., Gerstel, A., Leak, A.N., \& Smith, S.K. (2012). Patient provider preferences for survivorship care plan. Journal of Oncology Practice, 8(4), e80-86. doi:10.1200/JOP.2011.000401

McCorkle, R., Ercolano, E., Lazenby, M., Schulman-Green, D., Schilling, L.S., Lorig, K., \& Wagner, E.H. (2011). Self-Management: Enabling and empowering patients living with cancer as a chronic illness. Cancer journal for clinicians, 61(1), 50-62.

Meleis, A.I., Sawyer, L.M., Im, E.O., Hilfinger Messias, D.K. \& Schumacher, K. (2000). Experiencing transitions: An emerging middle-range theory. Advances in Nursing Science, 23(1), 12-28.

Miles, M.B., \& Huberman, A.M. (2003). Analyse des données qualitatives. (2nd ed.). Bruxelles: Groupe De Boeck.

Nicolaije, K.A., Husson, O., Ezendam, N.P., Vos, M.C., Kruitwagen, R.F., Lybeert, M.L., \& van de Poll-Franse, L.V. (2012). Endometrial cancer survivors are unsatisfied with receive information about diagnosis, treatment and follow-up: A study from the populationbased PROFILES registry. Patient Education and Counseling, 88(3), 427-435.

Osborne, R.H., Elsworth, G.R., \& Whitfield, K. (2007). The health Education Impact Questionnaire (heiQ): An outcomes and evaluation measure for patient education and self-management interventions for people with chronic conditions. Patient Education and Counseling, 66(2), 192-201.

Papadakos, J., Bussière-Côté, S., Abdelmutti, N., Catton, P., Friedman, A.J., Massey, C., Urowitz, S., \& Ferguson, S.E. (2012). Informational needs of gynecologic cancer survivors. Gynecologic Oncolog $\gamma$, 124(3) 452-457.

Patton M.Q. (1997). Utilization-focused evaluation: the new century text (3rd Ed.). Thousand Oaks, CA: Sage Publications.

Sabatino, S.A., Thompson, T.D., Smith, J.L., Rowland, J.H., Forsythe, L.P., Pollack, L., \& Hawkins, N.A. (2013). Receipt of cancer treatment summaries and follow-up instructions among adult cancer survivors: Results from a national survey. Journal of Cancer Survivorship, 7, 32-43.

Salani, R. (2013). Survivorship planning in gynecologic cancer patients. Gynecologic Oncology, 130(2), 389-397.

Simard, S., \& Savard, J. (2015). Screening and comorbidity of clinical levels of fear of cancer recurrence. Journal of Cancer Survivorship: Research and Practice, 9(3), 481-491. doi:10.1007/s1176401504244
Simard, S., Thewes, B., Humphris, G., Dixon, M., Hayden, C., Mireskandari, S., \& Ozakinci, G. (2013). Fear of cancer recurrence in adult cancer survivors: A systematic review of quantitative studies. Journal of Cancer Survivorship: Research and Practice, 7(3), 300-322.

Smith, S.L., Singh-Carlson, S., Downie, L., Payeur, N., \& Wai, E.S. (2011). Survivors of breast cancer: Patient perspectives on survivorship care planning. Journal of Cancer Survivorship: Research and Practice, 5(4), 337-344.

Société Canadienne du cancer. (2015). Statistiques canadiennes sur le cancer. Comité directeur de la Société Canadienne du cancer. Toronto, ON.

Sprague, B.L., Dittus, K.L., Pace, C.M., Dulko, D., Pollack, L.A., Hawkins, N.A., \& Geller, B.M. (2013). Patient satisfaction with breast and colorectal cancer survivorship care plans. Clinical Journal of Oncology Nursing, 17(3), 266-272.

Steele, R., \& Fitch, M.I. (2008). Supportive care needs of women with gynecologic cancer. Cancer Nursing, 31(4), 284-291.

Stricker, C.T., Jacobs, L.A., Risendal, B., Jones, A., Panzer, S., Ganz, P.A., ... Palmer, S.C. (2011). Survivorship care planning after the Institute of Medicine recommendations: How are we faring? Journal of Cancer Survivorship: Research and Practice, 5(4), 358-370. doi:10.1007/s1176401110964

Thorne, S.E., \& Stajduhar, K.I. (2012). Patient perceptions of communications on the threshold of cancer survivorship: implications for provider responses. Journal of Cancer Survivorship: Research and Practice, 6(2), 229-237.

Vaz, A.F., Conde, D.M., Costa-Paiva, L., Morais, S.S., Esteves, S.B., \& Pinto-Neto, A.M. (2011). Quality of life and adverse events after radiotherapy in gynecologic cancer survivors: A cohort study. Archives of Gynecology and Obstetrics, 284(6), 1523_1531.

Vollrath, M., Zenger, M., Singer, S., Einenkel, J., \& Hinz, A. (2013). The course of fatigue in patients with gynecologic and breast cancer. Journal of Gynecologic Oncology, 24(3), 280-286.

Wu, H.S., \& Harden, J.K. (2015). Symptom burden and quality of life in survivorship: A review of literature. Cancer Nursing, 38(1), E29-E54. 